University of Nebraska - Lincoln

DigitalCommons@University of Nebraska - Lincoln

Publications from USDA-ARS / UNL Faculty

U.S. Department of Agriculture: Agricultural

Research Service, Lincoln, Nebraska

2015

\title{
Female breakfast skippers display a disrupted cortisol rhythm and elevated blood pressure
}

\author{
Megan Witbracht \\ University of California, Davis \\ Nancy L. Keim \\ University of California, Davis \& USDA, ARS, Western Human Nutrition Research Center, Obesity and \\ Metabolism Research Unit, nancy.keim@ars.usda.gov \\ Shavawn Forester \\ Tahoe Forest Hospital \\ Adrianne Widaman \\ University of California, Davis \\ Kevin D. Laugero \\ University of California, Davis \& USDA, ARS, Western Human Nutrition Research Center, Obesity and \\ Metabolism Research Unit, kevin.laugero@ars.usda.gov
}

Follow this and additional works at: https://digitalcommons.unl.edu/usdaarsfacpub

Part of the Agriculture Commons

Witbracht, Megan; Keim, Nancy L.; Forester, Shavawn; Widaman, Adrianne; and Laugero, Kevin D., "Female breakfast skippers display a disrupted cortisol rhythm and elevated blood pressure" (2015). Publications from USDA-ARS / UNL Faculty. 2462.

https://digitalcommons.unl.edu/usdaarsfacpub/2462

This Article is brought to you for free and open access by the U.S. Department of Agriculture: Agricultural Research Service, Lincoln, Nebraska at DigitalCommons@University of Nebraska - Lincoln. It has been accepted for inclusion in Publications from USDA-ARS / UNL Faculty by an authorized administrator of DigitalCommons@University of Nebraska - Lincoln. 


\title{
Female breakfast skippers display a disrupted cortisol rhythm and elevated blood pressure
}

\author{
Megan Witbracht ${ }^{\mathrm{a}}$, Nancy L. Keim ${ }^{\mathrm{a}, \mathrm{b}}$, Shavawn Forester ${ }^{\mathrm{c}}$, Adrianne Widaman ${ }^{\mathrm{a}}$, Kevin Laugero ${ }^{\mathrm{a}, \mathrm{b}, *}$ \\ a Department of Nutrition, 1 Shields Ave., University of California, Davis, CA 95616, USA \\ ${ }^{b}$ U.S. Department of Agriculture, Agricultural Research Service, Western Human Nutrition Research Center, Obesity and Metabolism Research Unit, Davis, CA 95616, USA \\ c Tahoe Forest Hospital, 10121 Pine Ave, Truckee, CA 96161, USA
}

\section{H I G H L I G H T S}

- Breakfast skipping is associated with increased concentrations of free cortisol.

- Chronic breakfast skippers also displayed elevations in blood pressure.

- Changes in cortisol metabolism may lead to deleterious metabolic outcomes.

\section{A R T I C L E I N F O}

\section{Article history:}

Received 15 September 2014

Received in revised form 12 December 2014

Accepted 24 December 2014

Available online 27 December 2014

\section{Keywords:}

Cortisol

Breakfast skipping

Blood pressure

Cardiometabolic health

\begin{abstract}
A B S T R A C T
Chronic stress and over-activity in the hypothalamic-pituitary-adrenal (HPA) axis may link breakfast skipping and poor cardiometabolic health. Missing the first major meal of the day in rodents prolongs elevated circulating corticosterone at a time when it's normally decreasing. To extend these findings to humans, we hypothesized that habitual breakfast skippers would display a similar pattern of circulating cortisol and alterations in meal and stress-induced cortisol reactions. Normal weight to obese women aged 18-45 years old who were strictly defined as either breakfast skippers $(n=30)$ or breakfast eaters $(n=35)$ were invited to participate in our study. Normal breakfast habits were maintained for the entire study period and each participant attended 4 lab visits. Over the first 2 lab visits, body composition, fasting clinical chemistries, and self-reports of chronic stress were assessed. On each of 2 additional days (lab visits 3 and 4), salivary free cortisol was measured at home upon waking and at bedtime, and in the lab in response to a standard lunch, ad libitum afternoon snack buffet, and stress and control (relaxation) tasks. The order of the control and stress test visits was randomized. While body weight, body composition, HOMA-IR, total and HDL cholesterol did not statistically differ $(p>0.05)$, both diastolic and systolic blood pressure was elevated $(p<0.01)$ and LDL cholesterol was lower $(\mathrm{p}=0.04)$ in the breakfast skipper group. Compared to the breakfast eaters and on the control task visit only, breakfast skippers had higher circulating cortisol from arrival to midafternoon $(p<0.01)$ and during the snack buffet $(p<0.05)$. Furthermore, the lunch-induced cortisol reaction was larger in the 'skippers' $(p=0.03)$. On both stress and control visit days, the diurnal cortisol amplitude was significantly $(p=0.02)$ blunted in breakfast skippers. Self-reports of chronic stress did not differ between the groups. These data indicate that habitually skipping breakfast is associated with stress-independent over-activity in the HPA axis which, if prolonged, may increase risk (e.g., hypertension) for cardiometabolic disease in some people.
\end{abstract}

(C) 2014 Published by Elsevier Inc.

\section{Introduction}

Breakfast skipping is prevalent and occurs regularly in $20 \%$ of US adults [1]. Among young men, minorities and the economically disadvantaged, this phenomenon is even higher [2,3]. Habitual breakfast skipping may be detrimental to health [4] and has been linked to

* Corresponding author at: Stress Biology and Nutrition Research Lab, Obesity and Metabolism Research Unit, Western Human Nutrition Research Center, ARS/USDA, USA

E-mail address: kevin.laugero@ars.usda.gov (K. Laugero). elevated blood pressure [5], insulin resistance [6], and dyslipidemia [7]. While associations between breakfast skipping and BMI, or increased energy intake have been reported [1,8-10], recent evidence disputes these claims $[11,12]$. Differences in the physiological response to skipping breakfast may explain the apparent discrepancies. However, a physiological basis connecting regular breakfast skipping and poor metabolic health is unclear. Given the negative effects of chronically elevated cortisol on metabolic health [13-16], cortisol increases in response to skipping breakfast may, over time, promote risk for metabolic dysfunction. Persistent increases in cortisol are linked to 
hyperglycemia [17], insulin resistance [15], abdominal obesity [18], and high blood pressure [19].

In one of the few human studies looking at the frequency of breakfast intake and circulating cortisol concentrations, individuals eating breakfast every day had lower daytime mean salivary cortisol than those individuals who never ate breakfast [20]. In another study, breakfast skipping accounted for $22 \%$ of the inverse association between socioeconomic status and salivary cortisol [21]. In rodents, preventing consumption of the first meal of the day (lights off) significantly elevates corticosterone, which resolves when the animals are allowed to eat [22]. This phenomenon has not been well documented in humans.

We hypothesized that self-reported breakfast skippers would have elevated circulating cortisol, particularly during the morning and up until lunch. Secondly, we hypothesized cardiometabolic disease risk factors including insulin sensitivity, lipid profile, waist to hip circumference, and blood pressure will be higher in breakfast skippers, relative to breakfast eaters. Thirdly, we hypothesized that ongoing (chronic) stress may be more prominent in breakfast skippers and may contribute to elevated cortisol.

\section{Materials and methods}

\subsection{Participants and recruitment}

The Institutional Review Board at the University of California, Davis, approved the protocol. Based on the following criteria, women were invited to participate in a cross sectional observational study. Participants were premenopausal aged 18 to 45 years with a BMI of less than $40 \mathrm{~kg} / \mathrm{m}^{2}$, weight stable, maintained a typical diurnal schedule and were classified as either being "breakfast eaters" or "breakfast skippers." A breakfast eater was defined as someone who consumed at least $15 \%$ of their estimated total daily energy needs in solid food between the hours of $4 \mathrm{am}$ and $10 \mathrm{am}$, at least 6 days per week. A breakfast skipper was defined as someone who did not eat any solid food between the hours of 4 am and 10 am at least 4 days per week. Breakfast consumption is poorly defined in the literature therefore a strict set of criteria was developed to clearly separate the "skipper" group from the "eater" group [23-26]. A registered dietitian confirmed breakfast eating status using 3,24-hour recalls administered over the phone. Women were excluded if they used tobacco or chronic medications outside of oral contraceptives, were pregnant or lactating, or had a current diagnosis of any endocrine, metabolic or digestive disorders. Women were also excluded if they reported any past or current diagnosis of adrenal or pituitary specific disorders.

\subsection{Experimental procedures}

Eligible women were invited to participate in 4 visits at the Western Human Nutrition Research Center. At the first visit, a fasting blood sample was collected using standard phlebotomy, and height, weight and blood pressure were collected. Approximately 2 weeks later at visit 2 , height and weight measurements were repeated and body composition was measured. Subjects were also instructed on proper technique for saliva collection before completing several questionnaires related to stress and eating behavior. At the final two visits, which occurred approximately two months after visit 1 , volunteers participated in 6 hour lab protocols. These visits included a standard lunch which was designed to provide $35 \%$ of daily energy needs for each subject; an afternoon snack buffet and either a validated social stress test designed to elicit a cortisol response, or a similarly timed period of relaxation that functioned as control to the social stress test (Table 1). For the final two visits, order was randomized and each volunteer participated in both control and stress visits. Menstrual cycle was tracked throughout the study because cycle phase has been shown to affect the cortisol response to a stressor [27]. When possible, subjects participated in visits 3 and 4 during the follicular phase of their cycle.
Table 1

Test day ${ }^{\mathrm{a}}$ timeline schematic for a protocol administered twice ${ }^{\mathrm{b}}$ to a group of breakfast eaters and a group of breakfast skippers.

\begin{tabular}{|c|c|c|c|}
\hline Time & Saliva & Meals & Task \\
\hline Waking & $\swarrow$ & & \\
\hline Waking $+30 \mathrm{~min}$ & $\prec$ & & \\
\hline Variable & & Maintain usual breakfast habit & \\
\hline $1200 \mathrm{~h}$ & $\swarrow$ & & POMS \\
\hline $1230 \mathrm{~h}$ & $\prec$ & & \\
\hline $1235 \mathrm{~h}-1250 \mathrm{~h}$ & & Standard lunch ${ }^{\mathrm{c}}$ & \\
\hline $1315 \mathrm{~h}$ & 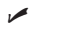 & & \\
\hline $1330 \mathrm{~h}$ & $\mathscr{}$ & & PSS \\
\hline $1400 \mathrm{~h}$ & $\swarrow$ & & POMS \\
\hline $1450 \mathrm{~h}$ & $\prec$ & & \\
\hline $1500 \mathrm{~h}-1520 \mathrm{~h}$ & & & TSST or Control \\
\hline $1525 \mathrm{~h}$ & $\swarrow$ & & POMS \\
\hline $1555 \mathrm{~h}$ & $\prec$ & & \\
\hline $1600-1630 \mathrm{~h}$ & & Snack buffet & \\
\hline $1630 \mathrm{~h}$ & $\prec$ & & POMS \\
\hline $1700 \mathrm{~h}$ & $\prec$ & & \\
\hline Variable & & Maintain usual dinner/snack habit ${ }^{\mathrm{c}}$ & \\
\hline Bedtime & $\prec$ & & \\
\hline
\end{tabular}

a The time interval between the first visit and the TSST or the Control test day visit was 60 days.

b The two test day visits varied only in the type of task administered at $1500 \mathrm{~h}$. Subjects were randomized to receive either the TSST or the control relaxation task first and a crossover design was implemented.

c Lunch menu items included a cheese quesadilla (full fat cheese, low fat cheese, tortilla), potato chips, canned peaches, maraschino cherries, tomato salsa and water; TERTER was determined using the Dietary Reference Intakes for adult women with a sedentary physical activity level assumed. Test meal energy and macronutrient composition were adjusted in 100 kilocalorie increments and consisted of 55\% kcal from carbohydrates, $15 \%$ kcal from protein, and 30\% kcal from fat. PSS, Perceived Stress Scale; POMS Profile of Mood States; TER Total Energy Requirement; TSST Trier Social Stress Test.

\subsubsection{Blood pressure}

Diastolic and systolic blood pressure was measured twice, $1 \mathrm{~min}$ apart, using the automated Dinamap 1846 (Critikon/GE). Measurements were taken after a 5-minute rest period, on the non-dominant arm while participants were seated.

\subsubsection{Anthropometry and body composition}

Height and weight were measured using a wall mounted stadiometer (Ayrton Stadiometer model S100; Prior Lake, MN) and an electronic scale (Scale-tronic model 6002; Wheaton, IL), respectively. Body mass index was calculated as $\mathrm{kg} / \mathrm{m}^{2}$. Hip and waist circumference was measured in the standing position against bare skin with the abdomen relaxed and the arms hanging at the sides using a spring-loaded non-metallic tape. Hip circumference was measured at the maximum protuberance of the buttocks while waist circumference was measured midway between the lateral lower rib margin and the iliac crest. Total fat mass and percent of total body lean and fat mass were assessed using air-displacement plethysomography by the Bod-Pod (Life Measurement Systems; Concord CA) with participants wearing tight fitting clothing and a swim cap.

\subsubsection{Questionnaires}

The Perceived Stress Scale (PSS) and the Wheaton Chronic Stress Inventory (WCS) were used to quantify the magnitude of chronic stress and loss of control experienced outside of the lab (e.g., at work and home) while the Profile of Mood States (POMS) was used to capture acute changes in mood and perceived stress at 4 times during the social stress test and the control day visit (Table 2) [28-30]. The PSS is a 10item questionnaire designed to assess the frequency (from "never" to "very often") of various general stressful experiences over the previous month period. The WCS Inventory evaluates the presence of specific, personal, financial and work-related stressors using a 3 point scale (from "not at all true" to "very true") for 51 items. The POMS questionnaires use 65 unique terms defining an emotional, or emotion related 
Table 2

List of questionnaires administered on the study.

\begin{tabular}{|c|c|c|c|}
\hline Questionnaire & Abbreviation & Test day (time-points) & Purpose \\
\hline Eating and Appraisal Due to Emotions and Stress ${ }^{a}$ & EADES & 2 (once) & Evaluates coping style and stress eating susceptibility \\
\hline Perceived Stress Survey & PSS & 3,4 (once) & Evaluates stress perception (previous 1 month) \\
\hline Wheaton Chronic Stress & WCS & 2 (once) & Evaluates stress perception (previous 6 months) \\
\hline Profiles of Mood States & POMS & 3,4 (four times) & Examines change in mood state across an event \\
\hline
\end{tabular}

a The EADES was analyzed for each factor: 1) Emotion and Stress-Related Eating, 2) Appraisal of Resources/Ability to Cope, and 3) Appraisal of Outside Stressors and Influences.

state to assess mood. The Eating and Appraisal Due to Emotions and Stress (EADES) questionnaire was used to determine relative susceptibility to stress eating and food related mechanisms for coping [31]. The 49 item questionnaire identifies 3 distinct factors related to stress perception and coping style, 1) Emotion and Stress-Related Eating, 2) Appraisal of Resources/Ability to Cope, and 3) Appraisal of Outside Stressors and Influences. The questionnaires were administered at the WHNRC to participants in the fasted state while they sat alone in a quiet room.

\subsubsection{Trier Social Stress Test Protocol (TSST)}

Chronic stress can manifest as either enhanced or diminished acute stress cortisol reactivity? Therefore, we applied the TSST to physiologically examine group differences in cortisol reactivity as an objective marker of chronic stress and possible reason for elevated basal cortisol in breakfast skippers. On the morning of the final two test days, participants were instructed to follow their usual breakfast habits; the breakfast skippers were asked to refrain from eating while the eaters were asked to consume their usual breakfast meal at home. Participants arrived at the WHNRC in the midday and were provided a standard lunch meal in a private room in the Metabolic Research Unit (MRU). The standard lunch was scaled to each subject at $35 \%$ of estimated energy requirements, based on the Dietary Reference Intake equation for normal, overweight and obese adult women [32]. Two hours after lunch, subjects participated in a 20 -minute validated psychosocial stress task called the Trier Social Stress Test (test visit) [33] or sat and watched a nature video (control visit). Briefly, the stress task consisted of 3, 5minute periods where subjects were instructed to give a speech, participate in a question and answer session, and perform a mental subtraction task in front of two trained examiners. Thirty minutes after completion of the TSST, subjects were provided with an ad libitum snack food buffet that contained foods of varying palatability and energy density. The foods were weighed in the metabolic kitchen prior to, and immediately after the 30-minute exposure period. Following the snack buffet on the "stress test day", the study coordinator explained the purpose of TSST protocol. Participants remained at the center for one additional hour until they were permitted to leave. Saliva was collected for the measurement of free cortisol at 13 times throughout each of the final two test days. The Control test visit was identical to the stress test visit except that the stress test was replaced by having participants watch a video of nature scenes set to classical music.

\subsection{Salivary cortisol}

Saliva samples were collected in salivettes (Salimetrics, LLC) and stored at $-80{ }^{\circ} \mathrm{C}$ for the measurement of free cortisol from waking to bedtime, but were focused around the expected response at waking, mealtimes and test periods (Table 1 ).

For measurement of circulating free cortisol, thawed saliva samples were vortexed briefly and centrifuged for $3000 \mathrm{rpm}$ for $15 \mathrm{~min}$ at room temperature.

Samples were assayed per manufacturer's instructions in duplicate using a high sensitivity enzyme immunoassay (Salimetrics LLC) on a BioTek Synergy 2 Multi-Mode Reader (BioTek Instruments Inc.). The accepted intra- and inter-assay variability as suggested by the manufacturer was set at $\leq 10 \%$ and $\leq 15 \%$, respectively. The salivary cortisol awakening response (CAR) was calculated by subtracting the sample taken 30 min post-waking from the waking sample. The diurnal salivary cortisol amplitude from morning to night was calculated by subtracting bedtime sample from the sample taken 30 min post-waking. The stress and control task response was calculated by subtracting the post-task cortisol value taken immediately after the test or control period from the pre-task value (Table 1 ). The total cortisol output for the control and stress visits was estimated by calculating area under the curve $\left(\mathrm{AUC}_{0}\right)$ for the 10 samples collected at the WHNRC during each visit.

\subsection{Blood}

Fasting blood was collected at the first visit in an SST vacutainer for the measurement of serum glucose, triglycerides, total cholesterol, HDL cholesterol and LDL cholesterol by the Clinical Laboratory Improvement Amendments (CLIA) certified Pathology Lab at the University of California, Davis Medical Center. Blood was also collected in an EDTA vacutainer for the measurement of insulin; plasma insulin was analyzed using a sandwich immunoassay (Meso Scale Discovery, Gaithersburg, MD). The HOMA-IR was calculated as [fasting plasma glucose $(\mathrm{mg} / \mathrm{dL}) \times$ serum fasting insulin $(\mathrm{mIU} / \mathrm{L}) / 405]$ [34]

\subsection{Statistics}

Statistical analyses were performed using SPSS22 software. The normal distribution of each variable was tested using the Shapiro-Wilk Test for Normality with the statistic set to 0.95 . All cortisol variables were log transformed, but data are presented as the untransformed mean \pm the standard deviation or standard error. One-way ANOVA was used to determine differences between the skipper and eater groups for BMI, fat mass, fat-free mass, waist circumference, blood pressure, and HOMAIR. ANCOVA with a repeated measures design was used to test for group and within subject differences in circulating cortisol across all sampling times collected in the lab and between lab visits. We also used a more targeted analysis of cortisol responsiveness; ANCOVA with repeated measures was applied to determine group and between visit differences in cortisol responses to waking, meals and the stress and control tasks. These responses included the cortisol awakening response (CAR), lunch- and buffet-induced cortisol, stress- and control task-induced cortisol, and the morning to bedtime cortisol amplitude. Because the saliva samples collected at home were less reliably monitored, the sample size reported for the CAR and amplitude are smaller. Previous evidence from animal studies [35] showed that, when rats are not allowed to eat their first meal of the day, basal concentrations of circulating cortisol remain elevated until food is ingested later in the day. Therefore, we also tested whether cortisol concentrations over the period extending from arrival at the WHNRC to the prestress/control task remained elevated in the breakfast skipper group. Since stress also affects cortisol, differences between breakfast skippers and eaters in perceived stress and stress related eating were tested using one-way ANOVA for total scores on Wheaton Chronic Stress, Perceived Stress, Profile of Moods Sates, and the Eating and Appraisal Due to Emotion and Stress. Age and menstrual cycle phase ( $\mathrm{n}=11$ in luteal phase) were used as covariates in all analyses. The pre-meal and prestress/control task cortisol concentrations were used as a covariate to statistically control for possible group differences in pre-meal and pre- 
stress/control task cortisol. To statistically adjust for possible group differences in the amount of food consumed on buffet-induced cortisol, caloric intake from the buffet was included in the ANCOVA model as a covariate. OC use has been associated with significant changes in free cortisol [27]. Three subjects used oral contraceptives (OC) during the study. Their inclusion in the final analyses did not change the results therefore their data was included.

\section{Results}

\subsection{Participants and anthropometric measures}

Sixty-five women ( $\mathrm{n}=35$ breakfast eaters, $\mathrm{n}=30$ breakfast skippers) completed all visits and were included in the final analyses. Body weight, BMI, fat mass, fat free mass, waist to hip ratio, total cholesterol, and HDL cholesterol measured at screening were not different between breakfast conditions ( $p>0.05$ ). However, LDL cholesterol was lower and systolic and diastolic blood pressures were higher in the skipper group ( $p=0.043, p=0.003, p=0.009$, respectively) (Table 3 ). Fasting glucose and HOMA-IR did not differ between groups ( $p>0.05$ ).

\subsection{Stimulated cortisol}

Our repeated measures analysis of cortisol showed a significant group $\times$ visit interaction $(p=0.02)$ and a group $\times$ time interaction $(p<0.01)$. Compared to breakfast eaters, average circulating cortisol concentrations across the test day (from arrival to the end of the test visit) were higher in the skipper group on the control day $(\mathrm{p}=0.01)$ (Fig. 1). On the control test day, the mean cortisol concentration for the three samples collected at the lunch and buffet meals (immediately prior to the meal, 30-45 min after, and 60 min after initiation of the meals) were higher ( $p_{\text {lunch }}=0.05$, $p_{\text {buffet }}<0.01$ ) in the skipper group. At the lunch meal only, the meal-induced cortisol reaction (post-meal samples relative to pre-meal) was higher $(p=0.03)$ in the skipper group at 30 and 60 min after lunch.

Table 3

Anthropometric, demographic, metabolic, and behavioral characteristics measured at screening of a group of routine breakfast eaters and a group of routine breakfast skippers.

\begin{tabular}{|c|c|c|c|}
\hline \multirow[t]{2}{*}{ Variable } & \multicolumn{2}{|c|}{ Breakfast habit } & \multirow[t]{2}{*}{ Significance $^{1}$} \\
\hline & $\begin{array}{l}\text { Eater } \\
(n=35)\end{array}$ & $\begin{array}{l}\text { Skipper } \\
(\mathrm{n}=30)\end{array}$ & \\
\hline Education $(\mathrm{y})$ & $15.8 \pm 0.9$ & $14.8 \pm 2.2$ & ns \\
\hline Body mass (kg) & $62.1 \pm 12.5$ & $67.0 \pm 18.7$ & ns \\
\hline $\operatorname{BMI}\left(\mathrm{kg} / \mathrm{m}^{2}\right)$ & $22.8 \pm 4.2$ & $24.8 \pm 6.7$ & ns \\
\hline Fat mass (\%) & $28.2 \pm 6.9$ & $30.2 \pm 9.1$ & ns \\
\hline Fat free mass (kg) & $43.4 \pm 6.5$ & $45.9 \pm 7.5$ & ns \\
\hline Systolic blood pressure (mm Hg) & $101.1 \pm 15.4$ & $111.8 \pm 11.4$ & 0.003 \\
\hline Diastolic blood pressure (mm Hg) & $63.4 \pm 8.5$ & $68.9 \pm 8.1$ & 0.009 \\
\hline Pulse (bpm) & $64.1 \pm 10.5$ & $68.0 \pm 9.0$ & ns \\
\hline Waist to hip ratio & $0.76 \pm 0.07$ & $0.74 \pm 0.07$ & ns \\
\hline Total cholesterol (mg/dL) & $176.6 \pm 24.4$ & $164.8 \pm 25.7$ & ns \\
\hline $\mathrm{LDL}(\mathrm{mg} / \mathrm{dL})$ & $102.6 \pm 19.0$ & $93.0 \pm 18.6$ & 0.043 \\
\hline $\mathrm{HDL}(\mathrm{mg} / \mathrm{dL})$ & $56.4 \pm 13.9$ & $56.9 \pm 14.5$ & ns \\
\hline Fasting glucose (mg/dL) & $83.3 \pm 6.5$ & $83.3 \pm 7.1$ & ns \\
\hline Fasting insulin (mIU/mL) & $9.8 \pm 6.4$ & $11.0 \pm 7.8$ & ns \\
\hline HOMA-IR & $2.0 \pm 1.5$ & $2.3 \pm 1.8$ & ns \\
\hline PSS & $17.2 \pm 6.8$ & $16.4 \pm 6.5$ & ns \\
\hline WCS & $15.1 \pm 7.9$ & $18.5 \pm 12.1$ & ns \\
\hline EADES factor 1 & $84.3 \pm 15.8$ & $90.8 \pm 19.5$ & ns \\
\hline EADES factor 2 & $76.4 \pm 7.2$ & $76.7 \pm 9.4$ & ns \\
\hline EADES factor 3 & $13.9 \pm 3.5$ & $14.8 \pm 3.9$ & ns \\
\hline
\end{tabular}

BMI, Body Mass Index; HOMA-IR, Homeostatic Model Assessment-Insulin Resistance; LDL, Low Density Lipoprotein; HDL, High Density Lipoprotein; WCS, Wheaton Chronic Stress Scale; PSS, Perceived Stress Scale; EADES Eating and Appraisal Due to Emotion and Stress.

${ }^{1}$ Different from breakfast skippers $(\mathrm{p}<0.05)$.

\subsection{Basal cortisol}

Basal, or non-stimulated concentrations of cortisol were also higher in the breakfast skipping group on the control test day from noon to $1500 \mathrm{~h}$, prior to watching the video $(\mathrm{p}<0.01)$. While the CAR was not different between groups, the morning to evening amplitude was smaller in the skipper group (Breakfast Eater, $\mathrm{n}=24$, Breakfast Skipper $\mathrm{n}=23$ ), regardless of the test day $(\mathrm{p}=0.02)$ (Fig. 2$)$. Scores on the WCS, PSS, POMS and the EADES were not different between the groups $(p>0.05)$ (Table 3). Cortisol reactivity to the stress test did not differ between breakfast skippers and eaters.

\section{Discussion}

The mechanisms linking breakfast skipping and poor health are unclear. Breakfast is typically consumed after an extended fast and after a period of sleep. It has been proposed that the typically elevated waking cortisol promotes arousal and drive to eat [36,37]. When breakfast is consumed, cortisol steadily drops. In meal-trained rats, if the first meal is missed, corticosterone ( primary glucocorticoid in rodents) concentrations remain elevated hours after it would typically fall $[22,35,38]$. Breakfast in humans or the first major meal of the day in rats likely resets activity in the HPA axis and reduces glucocorticoid concentrations. If this re-setting fails to occur, peak glucocorticoid concentrations persist for longer than normal over this period of the diurnal glucocorticoid rhythm. However, this has not been thoroughly examined in humans. Our results showed marked elevations of both non-stimulated and meal-stimulated cortisol concentrations in women who regularly skip breakfast more than 4 times a week.

Our results suggest that skipping breakfast diminished the typical drop in cortisol after eating breakfast and, therefore, maintained elevated cortisol throughout the morning and early afternoon. Given the role of glucocorticoids in supporting energy metabolism and food intake, it's not surprising that skipping the first major meal of the day would increase circulating cortisol concentrations. In the fasted state, or after skipping the first major meal of the day, the maintenance of relatively high cortisol concentrations promotes endogenous glucose production and ensures energy for organs such as the brain. In humans, glucocorticoid administration stimulates caloric intake [39]. In rats, glucocorticoid administration promotes the ingestion of energy dense, palatable food [40]. Similar results have been reported in humans exposed to a cortisol-inducing social stressor [41]. Therefore, sustained elevations in cortisol associated with skipping breakfast may function to enable use of endogenous energy in the short term and stimulate food intake in order to replenish lost energy after breakfast skipping. We did not detect differences between the two groups in energy or macronutrient consumption from the ad lib buffet. Others have reported a similar lack of association between food intake and breakfast skipping [42]. It is possible that, in a home or more natural setting, we would have found that breakfast skippers consume more calories later in the day to compensate for reduced consumption in the morning.

Food intake, particularly at a midday meal reliably increases circulating cortisol [43-45]. The magnitude of this response for women appears to depend on the protein content. Two meal types were used in this study to probe cortisol reactivity, a fixed calorie lunch meal and an ad libitum afternoon snack. The lunch meal contained $15 \%$ of its calories from protein and, as predicted, stimulated a measurable cortisol response in both skippers and eaters. However, this effect was larger in the breakfast skippers, but only during the control (non-stress task) visit. For the buffet associated cortisol response, mean intake of protein was only $6.6 \%$ and $6.2 \%$ of total calories from the buffet in eaters and skippers, respectively, but cortisol concentrations still increased in both groups during the buffet. However, the buffet related cortisol concentrations were higher in the breakfast skippers, and only on the control visit. We do not know why differences in meal-induced cortisol were specific to the control visit. Although we suspect that the 

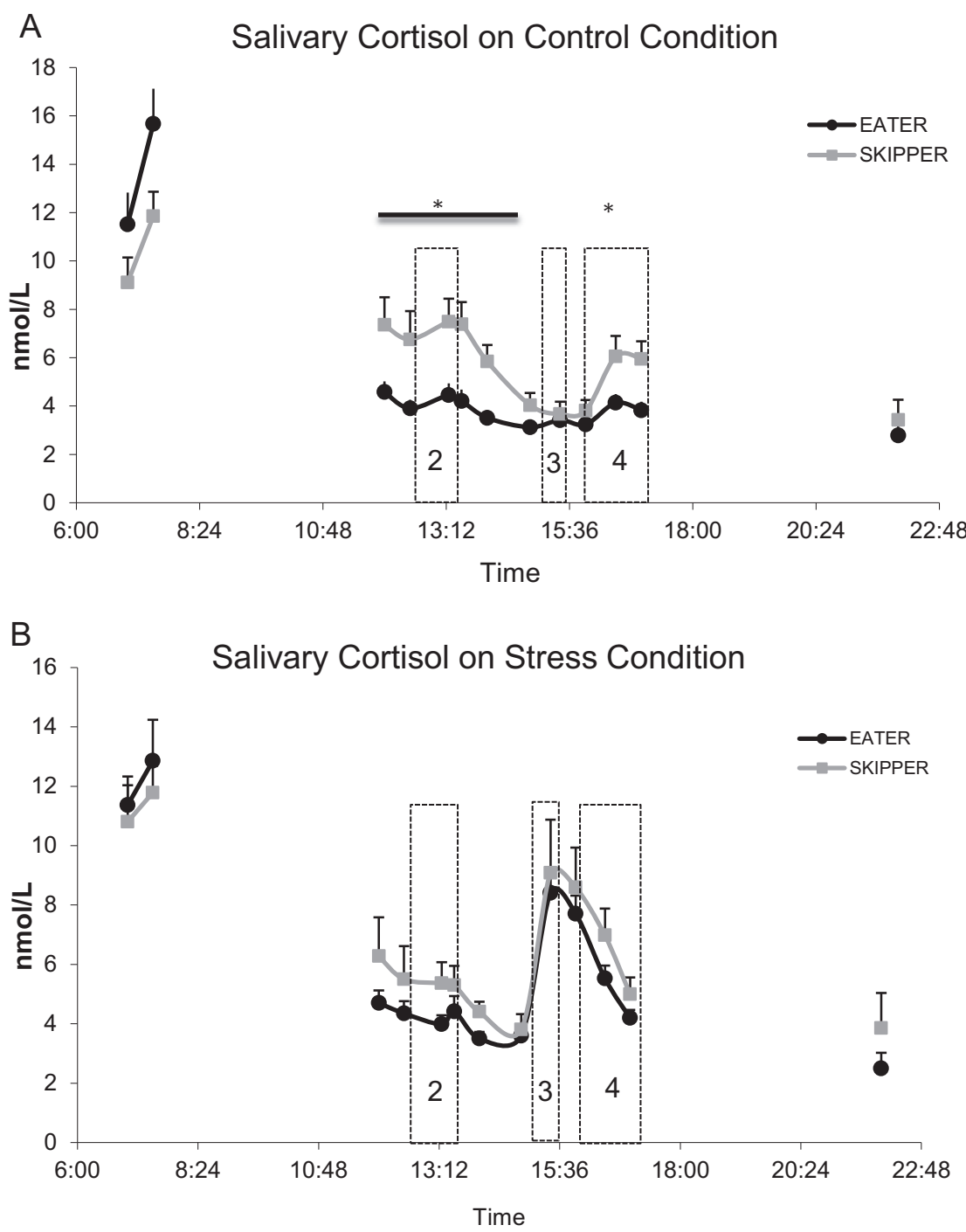

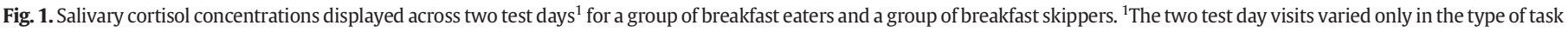

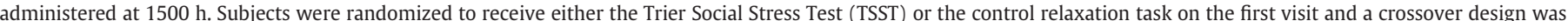

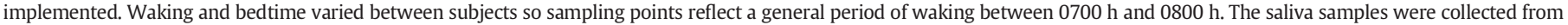

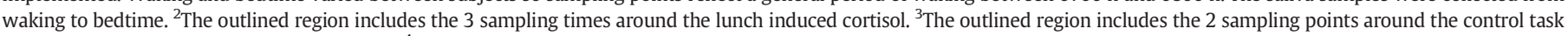

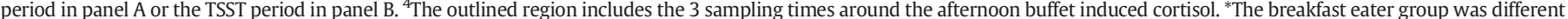

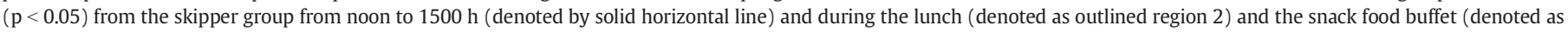
outlined region 4 ).

magnitude of the stress-induced cortisol response to have overwhelmed the buffet cortisol response, this does not explain the visitspecific effect for lunch-induced cortisol. Participants were not told in advance about the stress test, and the order in which women participated in stress or control visits was randomized. Furthermore, for both visits, the lunch and buffet offering were the same and presented in the same fashion. However, it is possible that some other more subtle factors not accounted for in this study explain these visitspecific effects.

Why did breakfast skippers have higher meal-associated cortisol? The exact function of the meal-induced cortisol response remains unclear, however glucocorticoids are known to play critical roles in glycemic control, substrate utilization and energy storage [46]. The cortisol response to a meal likely depends not only on the type and quantity of nutrients present in the gut, but also on the previous metabolic state. That is, if endogenous energy stores are diminished due to meal skipping, when food does become available and ingested, enhanced cortisol responsiveness to the meal may help promote energy storage as insurance against the possibility of future episodes of insufficient food intake. In the fed state, specifically in the presence of insulin, cortisol acts acutely to stimulate glycogen and fat storage for later use. Since cortisol also plays a role in promoting memory consolidation [47,48], mealassociated cortisol responses may have evolved to help the organism remember the relative time, quality, and location of a meal. This possible function of meal-induced cortisol may become especially critical when food is scarce or after a fast or skipped meal. Therefore, a more pronounced cortisol response to a meal after a period of fasting or meal skipping may result in order to ensure recollection of the time and location of the meal.

In addition to an increased cortisol tone, we found that breakfast skippers displayed a blunted or flat diurnal cortisol pattern. The diurnal cortisol rhythm in healthy individuals is typically defined by a high morning peak and a low evening nadir [49]. A flat or blunted diurnal amplitude is indicative of dysfunction in the HPA axis. This disrupted or more rigid cortisol pattern has been associated with work stress, Posttraumatic Stress Disorder, and cardiovascular disease risk, including high blood pressure, and is considered a hallmark of the hypercortisolemic Cushing's disease [50-52]. Interestingly, nutritive 


\section{Cortisol Amplitude}

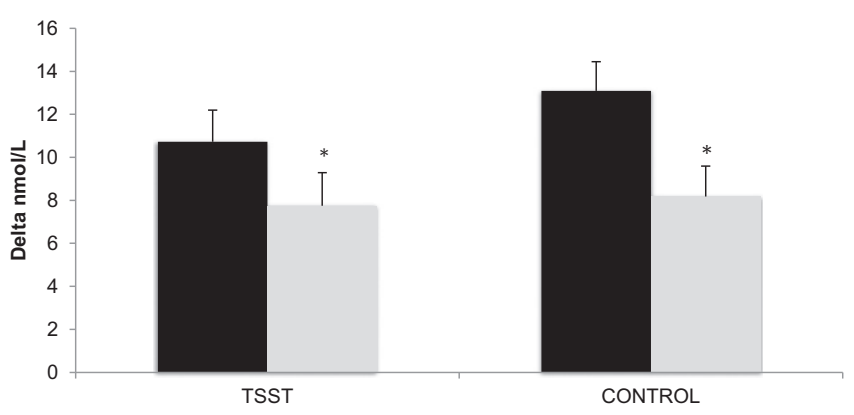

Fig. 2. The diurnal amplitude ${ }^{1}$ of salivary cortisol measured over two test days ${ }^{2}$ for a group of breakfast eaters and breakfast skippers. ${ }^{1}$ The diurnal amplitude was determined by subtracting the bedtime cortisol concentration from the cortisol concentration taken 30 min after waking. ${ }^{2}$ The two test day visits varied only in the type of task administered at $1500 \mathrm{~h}$. Subjects were randomized to receive either the Trier Social Stress Test (TSST) or the control relaxation task on the first visit and a crossover design was implemented. Waking and bedtime varied between subjects so sampling points reflect a general period of waking between $0700 \mathrm{~h}$ and $0800 \mathrm{~h}$. The saliva samples were collected from waking to bedtime. *The breakfast eater group was different from the skipper group $(\mathrm{p}<0.05)$.

status in the form of starvation or energy restriction has been shown to generate a similar diurnal cortisol pattern $[53,54]$.

Persistent elevations in cortisol coupled with a flat diurnal pattern are linked to cardiometabolic disease risk factors, such as insulin resistance [55], abdominal obesity [50], and high blood pressure [56,57]. In middle-aged men studied over a 16 -year period, breakfast skipping was associated with a $27 \%$ higher risk for developing cardiovascular disease [58]. We found that breakfast skippers had higher arterial blood pressure. As with cortisol, blood pressure [59] follows a diurnal rhythm, typically peaks in the morning around waking [60], and is acutely responsive to food intake; it typically decreases and then resolves within $3 \mathrm{~h}$ of consuming food [61]. Given that both cortisol and blood pressure follow a circadian rhythm and show acute responsivity to nutritive state, it is possible that persistent elevations in these measures resulted from the absence of a morning meal that would normally be required to help reset circadian driven processes. However, it is possible that cortisol and blood pressure might be elevated in breakfast skippers through independent mechanisms that may or may not be related to eating behavior or nutritive status.

Contrary to some $[62,63]$ and similar to other $[1,8,64,65]$ studies, we did not find that breakfast skippers had greater body weight or fat composition compared to breakfast eaters. Other factors, such as regular eating habits and number of days/years of breakfast skipping may determine whether breakfast skipping manifest into elevated body weight. It is difficult to reconcile our findings of lower LDL in breakfast skippers as it appears to contradict previous reports [7]. Overall, our findings support an association between breakfast skipping and cardiometabolic dysfunction [7,58,66-68], but also support elevated cortisol as a possible factor linking breakfast skipping and high blood pressure. However, prospective studies are needed to examine a mechanistic link between cortisol and high blood pressure in breakfast skippers.

Although subjects were asked to maintain their usual diet and exercise routines, we did not measure body weight on the final two test days when free cortisol was measured, we cannot rule out the effect that small changes in weight may have had on cortisol metabolism.

\section{Conclusion}

In conclusion, our results suggest that breakfast skippers have prolonged daily elevations in cortisol, which may explain higher blood pressure and other previously described cardiometabolic dysfunction in persons habitually skipping breakfast. We did not find other cardiometabolic disease risk factors to be elevated in breakfast skippers.
However, if this breakfast skipping along with altered cortisol activity persists, development of chronic disease is possible [67]. The women in our study were young ( $24.0 \pm 4.9$ years). Therefore, it is possible that, over time, other risk factors such as insulin resistance and elevated BMI would present in these breakfast skippers, as shown in other studies [6,66]. Given our findings, prospective mechanistic studies are warranted to determine the causal relationship between cortisol, breakfast skipping, and cardiometabolic health.

\section{Funding/support}

This research was supported with funding from USDA-CSREES NRI grant 2009-35215-05364 and USDA Agricultural Research Service intramural project number 5306-51530-019-00.

\section{Acknowledgments}

The USDA is an equal opportunity provider and employer.

\section{References}

[1] W.O. Song, et al., Is consumption of breakfast associated with body mass index in US adults? J. Am. Diet. Assoc. 105 (9) (2005) 1373-1382.

[2] A.M. Siega-Riz, B.M. Popkin, T. Carson, Differences in food patterns at breakfast by sociodemographic characteristics among a nationally representative sample of adults in the United States, Prev. Med. 30 (5) (2000) 415-424.

[3] W.O. Song, et al., Is consumption of breakfast associated with body mass index in US adults? J. Am. Diet. Assoc. 105 (9) (2005) 1373-1382.

[4] A.P. Smith, Breakfast cereal consumption and subjective reports of health by young adults, Nutr. Neurosci. 6 (1) (2003) 59-61.

[5] E.J. Kim, et al., Relationship between blood pressure parameters and pulse wave velocity in normotensive and hypertensive subjects: invasive study, J. Hum. Hypertens. 21 (2) (2007) 141-148.

[6] K.J. Smith, et al., Skipping breakfast: longitudinal associations with cardiometabolic risk factors in the Childhood Determinants of Adult Health Study, Am. J. Clin. Nutr. 92 (6) (2010) 1316-1325.

[7] G. Shafiee, et al., Association of breakfast intake with cardiometabolic risk factors, J. Pediatr. (Rio J.) 89 (6) (2013) 575-582.

[8] A.A. van der Heijden, et al., A prospective study of breakfast consumption and weight gain among U.S. men, Obesity (Silver Spring) 15 (10) (2007) 2463-2469.

[9] A.K. Kant, B.I. Graubard, A comparison of three dietary pattern indexes for predicting biomarkers of diet and disease, J. Am. Coll. Nutr. 24 (4) (2005) 294-303.

[10] U. Chitra, C.R. Reddy, The role of breakfast in nutrient intake of urban schoolchildren, Public Health Nutr. 10 (1) (2007) 55-58.

[11] T.A. Nicklas, C.E. O'Neil, G.S. Berenson, Nutrient contribution of breakfast, secular trends, and the role of ready-to-eat cereals: a review of data from the Bogalusa Heart Study, Am. J. Clin. Nutr. 67 (4) (1998) 757S-763S.

[12] J.M. Kerver, et al., Meal and snack patterns are associated with dietary intake of energy and nutrients in US adults, J. Am. Diet. Assoc. 106 (1) (2006) 46-53.

[13] L. Fardet, I. Petersen, I. Nazareth, Risk of cardiovascular events in people prescribed glucocorticoids with iatrogenic Cushing's syndrome: cohort study, BMJ 345 (2012) e4928.

[14] P. Bjorntorp, Neuroendocrine perturbations as a cause of insulin resistance, Diabetes Metab. Res. Rev. 15 (6) (1999) 427-441.

[15] C.J. Petersons, et al., Acute effect of increasing glucocorticoid replacement dose on cardiovascular risk and insulin sensitivity in patients with adrenocorticotrophin deficiency, J. Clin. Endocrinol. Metab. 99 (6) (2014) 2269-2276.

[16] A.S. DeSantis, et al., Associations of salivary cortisol levels with inflammatory markers: the Multi-Ethnic Study of Atherosclerosis, Psychoneuroendocrinology 37 (7) (2012) 1009-1018.

[17] T.S. Han, et al., Glucocorticoid treatment regimen and health outcomes in adults with congenital adrenal hyperplasia, Clin. Endocrinol. (Oxf.) 78 (2) (2013) 197-203.

[18] M.F. Dallman, et al., Glucocorticoids, the etiology of obesity and the metabolic syndrome, Curr. Alzheimer Res. 4 (2) (2007) 199-204.

[19] J.A. Whitworth, G.J. Mangos, J.J. Kelly, Cushing, cortisol, and cardiovascular disease, Hypertension 36 (5) (2000) 912-916.

[20] A.P. Smith, Stress, breakfast cereal consumption and cortisol, Nutr. Neurosci. 5 (2) (2002) 141-144.

[21] M.J. Dorsey, et al., Assessment of adrenal suppression in children with asthma treated with inhaled corticosteroids: use of dehydroepiandrosterone sulfate as a screening test, Ann. Allergy Asthma Immunol. 97 (2) (2006) 182-186.

[22] K.I. Honma, S. Honma, T. Hiroshige, Critical role of food amount for prefeeding corticosterone peak in rats, Am. J. Physiol. 245 (3) (1983) R339-R344.

[23] S. Cho, et al., The effect of breakfast type on total daily energy intake and body mass index: results from the Third National Health and Nutrition Examination Survey (NHANES III), J. Am. Coll. Nutr. 22 (4) (2003) 296-302.

[24] K. Vagstrand, et al., Eating habits in relation to body fatness and gender in adolescents-results from the 'SWEDES' study, Eur. J. Clin. Nutr. 61 (4) (2007) 517-525.

[25] P.S. Haines, D.K. Guilkey, B.M. Popkin, Trends in breakfast consumption of US adults between 1965 and 1991, J. Am. Diet. Assoc. 96 (5) (1996) 464-470. 
[26] A.M. Siega-Riz, B.M. Popkin, T. Carson, Trends in breakfast consumption for children in the United States from 1965-1991, Am. J. Clin. Nutr. 67 (4) (1998) 748S-756S.

[27] C. Kirschbaum, et al., Impact of gender, menstrual cycle phase, and oral contraceptives on the activity of the hypothalamus-pituitary-adrenal axis, Psychosom. Med. 61 (2) (1999) 154-162

[28] D. McNair, M. Lorr, L. Droppleman, Profile of Mood States, in: E.a.IT. Service (Ed.), 1992 (San Diego).

[29] S. Levenstein, et al., Development of the Perceived Stress Questionnaire: a new too for psychosomatic research, J. Psychosom. Res. 37 (1) (1993) 19-32.

[30] R.J.a.W.B. Turner, Checklist measurement of stressful life events, in: S. Cohen, R Kessler, L. Underwood Gordon (Eds.),Measuring Stress, 1995, pp. 29-58.

[31] A.D. Ozier, et al., The Eating and Appraisal Due to Emotions and Stress (EADES) Questionnaire: development and validation, J. Am. Diet. Assoc. 107 (4) (2007) 619-628.

[32] Institute of Medicine (U.S.), Panel on Macronutrients and Institute of Medicine (U.S.), Standing Committee on the Scientific Evaluation of Dietary Reference Intakes, Dietary Reference Intakes for Energy, Carbohydrate, Fiber, Fat, Fatty Acids, Cholesterol, Protein, and Amino AcidsNational Academies Press, Washington, D.C., 2005 (xxv, $1331 \mathrm{pp}$.).

[33] C. Kirschbaum, K.M. Pirke, D.H. Hellhammer, The 'Trier Social Stress Test'-a tool for investigating psychobiological stress responses in a laboratory setting, Neuropsychobiology 28 (1-2) (1993) 76-81.

[34] D.R. Matthews, et al., Homeostasis model assessment: insulin resistance and betacell function from fasting plasma glucose and insulin concentrations in man Diabetologia 28 (7) (1985) 412-419.

[35] M.F. Dallman, et al., The neural network that regulates energy balance is responsive to glucocorticoids and insulin and also regulates HPA axis responsivity at a site proximal to CRF neurons, Ann. N. Y. Acad. Sci. 771 (1995) 730-742.

[36] M. Follenius, et al., Nocturnal cortisol release in relation to sleep structure, Sleep 15 (1) (1992) 21-27.

[37] A. Clow, et al., The cortisol awakening response: more than a measure of HPA axis function, Neurosci. Biobehav. Rev. 35 (1) (2010) 97-103.

[38] S.B. Yoo, et al., Ingestion of non-caloric liquid diet is sufficient to restore plasma corticosterone level, but not to induce the hypothalamic c-Fos expression in fooddeprived rats, Nutr. Neurosci. 10 (5-6) (2007) 261-267.

[39] P.A. Tataranni, et al., Effects of glucocorticoids on energy metabolism and food intake in humans, Am. J. Physiol. 271 (2 Pt 1) (1996) E317-E325.

[40] S. Bhatnagar, et al., Corticosterone facilitates saccharin intake in adrenalectomized rats: does corticosterone increase stimulus salience? J. Neuroendocrinol. 12 (5) (2000) 453-460.

[41] E. Epel, et al., Stress may add bite to appetite in women: a laboratory study of stressinduced cortisol and eating behavior, Psychoneuroendocrinology 26 (1) (2001) 37-49.

[42] D.A. Levitsky, C.R. Pacanowski, Effect of skipping breakfast on subsequent energy intake, Physiol. Behav. 119 (2013) 9-16.

[43] E.L. Gibson, et al., Increased salivary cortisol reliably induced by a protein-rich midday meal, Psychosom. Med. 61 (2) (1999) 214-224.

[44] S.G. Lemmens, et al., Influence of consumption of a high-protein vs. highcarbohydrate meal on the physiological cortisol and psychological mood response in men and women, PLoS One 6 (2) (2011) e16826.

[45] M.J. Martens, et al., Effects of single macronutrients on serum cortisol concentrations in normal weight men, Physiol. Behav. 101 (5) (2010) 563-567.

[46] D.G.G.a.D. Shoback, Greenspan's Basic \& Clinical Endocrinology, 8e, McGraw-Hill, 2007.

[47] B. Roozendaal, Systems mediating acute glucocorticoid effects on memory consolidation and retrieval, Prog. Neuropsychopharmacol. Biol. Psychiatry 27 (8) (2003) 1213-1223.
[48] O.T. Wolf, HPA axis and memory, Best Pract. Res. Clin. Endocrinol. Metab. 17 (2) (2003) 287-299.

[49] G.W. Liddle, Analysis of circadian rhythms in human adrenocortical secretory activity, Arch. Intern. Med. 117 (6) (1966) 739-743.

[50] R. Rosmond, M.F. Dallman, P. Bjorntorp, Stress-related cortisol secretion in men: relationships with abdominal obesity and endocrine, metabolic and hemodynamic abnormalities, J. Clin. Endocrinol. Metab. 83 (6) (1998) 1853-1859.

[51] S.R. Kunz-Ebrecht, C. Kirschbaum, A. Steptoe, Work stress, socioeconomic status and neuroendocrine activation over the working day, Soc. Sci. Med. 58 (8) (2004) $1523-1530$

[52] T.B. Carroll, J.W. Findling, The diagnosis of Cushing's syndrome, Rev. Endocr. Metab. Disord. 11 (2) (2010) 147-153.

[53] I.M. Anderson, et al., The effect of moderate weight loss on overnight growth hormone and cortisol secretion in healthy female volunteers, J. Affect. Disord. 16 (2-3) (1989) 197-202

[54] M.G. Witbracht, et al., Dairy food consumption and meal-induced cortisol response interacted to influence weight loss in overweight women undergoing a 12-week, meal-controlled, weight loss intervention, J. Nutr. 143 (1) (2013) 46-52.

[55] C.S. Tam, et al., No effect of caloric restriction on salivary cortisol levels in overweight men and women, Metabolism 63 (2) (2014) 194-198.

[56] R. Rosmond, P. Bjorntorp, Blood pressure in relation to obesity, insulin and the hypothalamic-pituitary-adrenal axis in Swedish men, J. Hypertens. 16 (12 Pt 1) (1998) 1721-1726.

[57] S.B. Park, et al., Association of cortisol and the metabolic syndrome in Korean men and women, J. Korean Med. Sci. 26 (7) (2011) 914-918.

[58] L.E. Cahill, et al., Prospective study of breakfast eating and incident coronary heart disease in a cohort of male US health professionals, Circulation 128 (4) (2013) 337-343.

[59] J.M. Neutel, D.H. Smith, The circadian pattern of blood pressure: cardiovascular risk and therapeutic opportunities, Curr. Opin. Nephrol. Hypertens. 6 (3) (1997) 250-256.

[60] M.H. Smolensky, et al., Circadian rhythmic aspects of human cardiovascular function: a review by chronobiologic statistical methods, Chronobiologia 3 (4) (1976) 337-371.

[61] N.L. Smith, et al., The association between time since last meal and blood pressure in older adults: the cardiovascular health study, J. Am. Geriatr. Soc. 51 (6) (2003) 824-828.

[62] L.M. Kent, A. Worsley, Breakfast size is related to body mass index for men, but not women, Nutr. Res. 30 (4) (2010) 240-245.

[63] B. Abalkhail, S. Shawky, Prevalence of daily breakfast intake, iron deficiency anaemia and awareness of being anaemic among Saudi school students, Int. J. Food Sci. Nutr. 53 (6) (2002) 519-528.

[64] E. Kapantais, et al., Breakfast skipping and its relation to BMI and healthcompromising behaviours among Greek adolescents, Public Health Nutr. 14 (1) (2011) 101-108.

[65] L. Azadbakht, et al., Breakfast eating pattern and its association with dietary quality indices and anthropometric measurements in young women in Isfahan, Nutrition 29 (2) (2013) 420-425.

[66] P. Deshmukh-Taskar, et al., The relationship of breakfast skipping and type of breakfast consumed with overweight/obesity, abdominal obesity, other cardiometabolic risk factors and the metabolic syndrome in young adults. The National Health and Nutrition Examination Survey (NHANES): 1999-2006, Public Health Nutr. 16 (11) (2013) 2073-2082.

[67] F. Kobayashi, et al., Effect of breakfast skipping on diurnal variation of energy metabolism and blood glucose, Obes. Res. Clin. Pract. 8 (3) (2014) e201-e298.

[68] S. Reutrakul, et al., The relationship between breakfast skipping, chronotype, and glycemic control in type 2 diabetes, Chronobiol. Int. 31 (1) (2014) 64-71. 